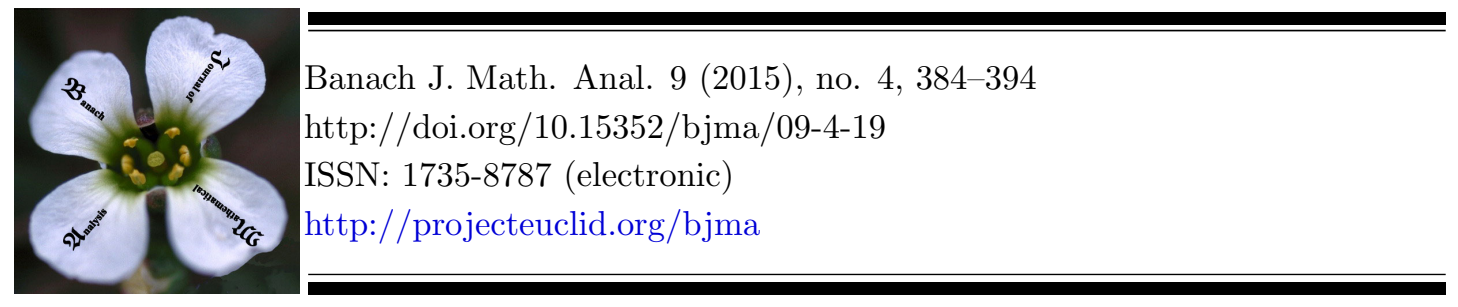

\title{
A NOTE ON INDEX-PROPER MULTISPLITTINGS OF MATRICES
}

\author{
ALEKHA KUMAR BALIARSINGH ${ }^{1}$ AND LITISMITA JENA ${ }^{2} *$ \\ Communicated by P. Y. Wu
}

\begin{abstract}
Matrix splitting and its convergence theorems are useful tools for finding solution of linear system of equations, iteratively. In this article, we introduce a few matrix splittings arising from index-proper splittings. Then their convergence results and their applications to multisplitting theory are studied.
\end{abstract}

\section{INTRODUCTION AND PRELIMINARIES}

We use the symbol $\mathbb{R}$ for the set of real numbers. The set of all $m \times n$ matrices over $\mathbb{R}$ is denoted by $\mathbb{R}^{m \times n}$. As usual, let $R(A)$ be the range space and $N(A)$ the null space of $A \in \mathbb{R}^{m \times n}$. If $L, M$ are subspaces of $R^{n}$, the projection onto $L$ along $M$ is denoted by $P_{L, M}$. So, $P_{L, M} B=B$ if and only if $R(B) \subseteq L$, and $B P_{L, M}=B$ if and only if $N(B) \subseteq M$. The symbol $\oplus$ is used for the direct sum of vector spaces. We denote the transpose of a matrix $A$ by $A^{T}$ and rank of a matrix $A$ by $r(A)$. Then, a matrix $A$ is said to be non-negative (i.e., $A \geq 0$ ) if all the entries of $A$ are non-negative, and $B \geq C$ for matrices $B$ and $C$, if $B-C \geq 0$. We also use these notation and nomenclature for vectors. The spectral radius of a square matrix $A$ is denoted by $\rho(A)$, and is equal to the maximum of the moduli of the eigenvalues of $A$.

Date: Received: Sep. 1, 2014; Revised: Dec. 2, 2014; Accepted: Mar. 17, 2015.

* Corresponding author.

2010 Mathematics Subject Classification. Primary 15A09; Secondary 65F15, 65F20.

Key words and phrases. Drazin inverse, group inverse, non-negativity, index-proper splittings, convergence theorem, comparison theorem. 
The Moore-Penrose inverse of a matrix $A \in \mathbb{R}^{m \times n}$, denoted by $A^{\dagger}$ is the unique solution $X$ of the equations

$$
A X A=A, X A X=X,(A X)^{T}=A X, \text { and }(X A)^{T}=X A .
$$

The index of $A \in \mathbb{R}^{n \times n}$ is the least non-negative integer $k$ such that $R\left(A^{k+1}\right)=R\left(A^{k}\right)$, and we denote it by $\operatorname{ind}(A)$. Then $\operatorname{ind}(A)=k$ if and only if $R\left(A^{k}\right) \oplus N\left(A^{k}\right)=\mathbb{R}^{n}$. Also, for $l \geq k, R\left(A^{l}\right)=R\left(A^{k}\right)$ and $N\left(A^{l}\right)=N\left(A^{k}\right)$. Of course, $k=\operatorname{ind}(A)$ is the dimension of the largest Jordan block with eigenvalue zero. In particular, $\operatorname{ind}(A)=0$ if $\mathrm{A}$ is a nonsingular matrix. The Drazin inverse of a matrix $A \in \mathbb{R}^{n \times n}$ is the unique solution $X \in \mathbb{R}^{n \times n}$ satisfying the equations:

$$
X A X=X, A X=X A \text {, and } A^{k+1} X=A^{k} \text {, where } k=\operatorname{ind}(A) .
$$

It is denoted by $A^{D}$. In general, the Drazin inverse can be expressed explicitly in terms of the Jordan canonical form of $A$ :

$$
A=S\left[\begin{array}{cc}
J & O \\
O & N
\end{array}\right] S^{-1} ; A^{D}=S\left[\begin{array}{cc}
J^{-1} & O \\
O & O
\end{array}\right] S^{-1},
$$

where $J$ contains the Jordan blocks corresponding to nonzero eigenvalues, and $N$ is nilpotent with $N^{k}=0$ and $N^{k-1} \neq 0$. With this representation of $A^{D}$, we can have $R\left(A^{k}\right)=R\left(A^{D}\right) ; N\left(A^{k}\right)=N\left(A^{D}\right)$ and hence $A A^{D}=P_{R\left(A^{k}\right), N\left(A^{k}\right)}=A^{D} A$. In particular, if $x \in R\left(A^{k}\right)$ then $x=A^{D} A x$. When $k=1$, then Drazin inverse is said to be group inverse and is denoted by $A^{\#}$. While Drazin inverse exists for all matrices, the group inverse does not. $A^{\#}$ exists if and only if $R(A) \cap N(A)=\{0\}$. If $A$ is nonsingular, then of course, we have $A^{-1}=A^{D}=A^{\#}=A^{\dagger}$. $A$ is said to be semi-monotone and Drazin monotone if $A^{\dagger} \geq 0$ and $A^{D} \geq 0$, respectively. Similarly, $A$ is group monotone if $A^{\#}$ exists and $A^{\#} \geq 0$.

A real square matrix $A$ is called monotone if $A x \geq 0 \Rightarrow x \geq 0$. Collatz [8] has shown that a matrix is monotone if and only if it is nonsingular and the inverse is non-negative. The notion of monotonicity has been generalized along several directions. An extension of the notion of monotonicity for characterizing nonnegativity of left inverses seems to have been first accomplished by Mangasarian [13]. Berman and Plemmons (Theorem 1, [3] and Theorem 2, [4]) proved the following characterizations for semi-monotone and group monotone matrices. $A$ is semi-monotone if and only if $A x \in \mathbb{R}_{+}^{n}+N\left(A^{T}\right)$ and $x \in R\left(A^{T}\right)$ imply $x \geq 0$. $A$ is group monotone if and only if $A x \in \mathbb{R}_{+}^{n}+N(A)$ and $x \in R(A)$ imply $x \geq 0$. Thereafter, Pye (Theorem 3, [15]) established that $A$ is Drazin monotone if and only if $A x \in \mathbb{R}_{+}^{n}+N\left(A^{k}\right)$ and $x \in R\left(A^{k}\right)$ imply $x \geq 0$. (See the book [1] for more details and the references cited therein for examples of applications of non-negative generalized inverses that include Numerical Analysis and linear economic models.) In this article, we are going to present characterizations of Drazin monotone matrices using matrix splitting theory.

The theory of matrix splittings is decisive for finding solutions of linear systems, and is also used for showing non-negativity of different generalized inverses. Recently, Jena and Mishra, [9] obtained many results for non-negativity of the Drazin inverse using several new matrix splittings. Applications of the Drazin inverse lie in many areas such as singular differential and difference equations, 
Markov chain, cryptography, iterative methods, multi-body dynamics and optimal control. Therefore, computation of the Drazin inverse and study of its properties have been an area of active research. Here only a few articles on the Drazin inverse are mentioned, but there is a vast amount of literature on it. (See the references $[9,11,17]$ and the references cited therein.)

In this paper, we plan to extend theory of regular and weak regular splittings(see Section 3 for these definitions) for singular square matrices using the Drazin inverse. We next aim to study convergence of such splittings and to apply to the theory of multisplittings.

The paper is organized as follows. In Section 2, we give a short repetition of well-known facts about index-proper splitting and some results related to the notion of non-negative matrices. The main results will be developed in Section 3 followed by a section called conclusions.

\section{INDEX-PROPER SPLITTING}

A splitting ${ }^{1} A=U-V$ is called an index-proper splitting ([9]) of $A \in \mathbb{R}^{n \times n}$ if $R\left(A^{k}\right)=R\left(U^{k}\right)$ and $N\left(A^{k}\right)=N\left(U^{k}\right)$, where $k=i n d(A)$. It reduces to index splitting $([17])$ if $\operatorname{ind}(U)=1$. When $k=1$, then an index-proper splitting becomes a proper splitting ([2]). Index-proper splittings lead to the iteration schemes:

$$
x^{i+1}=U^{D} V x^{i}+U^{D} b, i=0,1,2, \cdots
$$

and

$$
Y^{j+1}=U^{D} V Y^{j}+U^{D}, j=0,1,2, \cdots .
$$

For an index-proper splitting, the spectral radius of $U^{D} V$ is strictly less than 1 if and only if the above schemes converge to $A^{D} b$ and $A^{D}$, respectively to the system $A x=b$ (see [9]). More on index-proper splittings can be found in the recent articles $[9,11]$ and a simple method for constructing proper splittings can be found in [5]. Let us revisit some earlier results on index-proper splittings.

Theorem 2.1. (Theorem 3.2, [9]) Let $A=U-V$ be an index-proper splitting. Then

(a) $A A^{D}=U U^{D}=A^{D} A$;

(b) $I-U^{D} V$ is invertible;

(c) $A^{D}=\left(I-U^{D} V\right)^{-1} U^{D}$.

Lemma 2.2. (Lemma 1.8, [11]) $A=U-V$ be an index-proper splitting of real $n \times n$ matrix $A$. Let $\mu_{i}, 1 \leq i \leq s$ and $\lambda_{j}, 1 \leq j \leq s$ be the eigenvalues of $U^{D} V$ and $A^{D} V$, respectively. Then for every $j$, we have $1+\lambda_{j} \neq 0$. Also, for every $i$, there exists $j$ such that $\mu_{i}=\frac{\lambda_{j}}{1+\lambda_{j}}$ and for every $j$, there exists $i$ such that $\lambda_{j}=\frac{\mu_{i}}{1-\mu_{i}}$.

The following results will be used to prove the main results, and the first one is a part of a well-known theorem called Perron-Frobenius theorem.

\footnotetext{
${ }^{1} \mathrm{~A}$ splitting of a real square matrix $A$ is an expression of the form $A=U-V$, where $U$ and $V$ are matrices of the same order as of $A$
} 
Theorem 2.3. (Theorem 2.20, [14]) Let $A$ be a real square non-negative matrix. Then $A$ has a non-negative real eigenvalue equal to its spectral radius.

Theorem 2.4. (Theorem 2.21, [14]) If $A \geq B \geq 0$, then $\rho(A) \geq \rho(B)$.

Lemma 2.5. (Corollary 3.2, [6]) Let $B$ be a real square matrix. Let $B \geq 0$ and $x \geq 0$ be such that $B x-\alpha x \geq 0$. Then $\alpha \leq \rho(B)$.

Theorem 2.6. (Theorem 3.16, [14]) Let $X$ be a real square matrix such that $X \geq$ 0. Then $\rho(X)<1$ if and only if $(I-X)^{-1}$ exists and $(I-X)^{-1}=\sum_{k=0}^{\infty} X^{k} \geq 0$.

We conclude this section with the following lemma. (For more details, refer the article [2].)

Lemma 2.7. (Lemma 1, [2]) Let $K$ be a full cone in $\mathbb{R}^{n}$ and let $\left\{s_{i}\right\}_{i=0}^{\infty}$ be a $K$ monotone non-decreasing sequence. Let $t \in \mathbb{R}^{n}$ be such that $t-s_{i} \in K$ for every positive integer $i$. Then the sequence $\left\{s_{i}\right\}_{i=0}^{\infty}$ converges.

\section{Main Results}

The theory of splittings for square nonsingular matrices and its relationship with the solution of linear system equation is quite well-known. Standard iterative methods like the Jacobi, Gauss-Seidel and successive over-relaxation methods for solving a square nonsingular system of linear equations $A x=b$, arise from different choices of real square matrices $U$ and $V$, where $A=U-V$ and $b$ is a real $n$-vector. The book by Varga [14] contains several splittings such as regular and weak regular splittings. A splitting $A=U-V$ of a real square nonsingular matrix $A$ is

(i) regular splitting if $U^{-1}$ exists, $U^{-1} \geq 0$ and $V \geq 0$ ([14]),

(ii) weak regular splitting if $U^{-1}$ exists, $U^{-1} \geq 0$ and $U^{-1} V \geq 0([1,14])$,

The above authors also have shown that the regular and weak regular splitting of a monotone matrix is a convergent splitting (means that the spectral radius of the iteration matrix is less than 1). Jena et. al, [10] then extended these splittings to rectangular matrices and call it as proper regular splitting (see Definition 1.1, [10]). Then they have presented some convergence and comparison results for this splitting. Before these extensions, Jena and Mishra, [9] also proposed another extension of regular splitting which is presented next.

Definition 3.1. (Definition 4.13, [9]) A splitting $A=U-V$ of $A \in \mathbb{R}^{n \times n}$ is called $D$-regular splitting if it is an index-proper splitting satisfying the conditions $U^{D} \geq 0$ and $V \geq 0$.

In case of nonsingular matrices, the above definition coincides with the definition of a regular splitting and the results obtained below merge with the same results. We call D-regular splitting as index-proper regular splitting being motivated by the name of proper regular splitting. Hereon-words, all our matrices are real square matrices of order $n$. We now present a convergence theorem for the above splitting. 
Theorem 3.2. Let $A=U-V$ be an index-proper regular splitting of $A$. If $A^{D} \geq 0$, then

(a) $A^{D} \geq U^{D}$;

(b) $\rho\left(A^{D} V\right) \geq \rho\left(U^{D} V\right)$;

(c) $\rho\left(U^{D} V\right)=\frac{\rho\left(A^{D} V\right)}{1+\rho\left(A^{D} V\right)}<1$.

Proof. Given that $A=U-V$ is an index-proper regular splitting and $A^{D} \geq 0$. So we have $A=U-V$ be an index-proper splitting with $U^{D} \geq 0$ and $V \geq 0$.

(a) The fact $A=U-V$ is an index-proper splitting yields $A^{D}=\left(I-U^{D} V\right)^{-1} U^{D}$ so that $U^{D}=\left(I-U^{D} V\right) A^{D}$. Therefore $A^{D}-U^{D}=U^{D} V A^{D}$, i.e., $A^{D} \geq U^{D}$.

(b) Post-multiplying $V$ to $A^{D} \geq U^{D}$, and then by Theorem 2.4, we get $\rho\left(A^{D} V\right) \geq$ $\rho\left(U^{D} V\right)$.

(c) We have $A^{D} V \geq 0$. Let $\lambda$ be any eigenvalue of $A^{D} V$. Let $f(\lambda)=\frac{\lambda}{1+\lambda}, \lambda \geq 0$. Then $f$ is a strictly increasing function. Let $\mu$ be any eigenvalue of $U^{D} V$. Then by Lemma $2.2 \mu=\frac{\lambda}{1+\lambda}$. So, $\mu$ attains its maximum when $\lambda$ is maximum. But $\lambda$ is maximum when $\lambda=\rho\left(A^{D} V\right)$. As a result, the maximum value of $\mu$ is $\rho\left(U^{D} V\right)$. Hence $\rho\left(U^{D} V\right)=\frac{\rho\left(A^{D} V\right)}{1+\rho\left(A^{D} V\right)}<1$.

The example given below demonstrates the above result.

Example 3.3. Let $A=\left(\begin{array}{llll}0 & 0 & 1 & 1 \\ 0 & 5 & 0 & 0 \\ 0 & 0 & 5 & 0 \\ 0 & 0 & 0 & 0\end{array}\right)$ and $U=\left(\begin{array}{cccc}0 & 0 & 2 & 2 \\ 0 & 10 & 0 & 0 \\ 0 & 0 & 10 & 0 \\ 0 & 0 & 0 & 0\end{array}\right)$. The index of $A$ and $U$ are 2. Also $R\left(A^{2}\right)=R\left(U^{2}\right)$ and $N\left(A^{2}\right)=N\left(U^{2}\right)$. Hence $A=U-V$ is an index-proper splitting, where $V=U-A \geq 0$. Now $U^{D}=$ $\left(\begin{array}{cccc}0 & 0 & 1 / 50 & 0 \\ 0 & 1 / 10 & 0 & 0 \\ 0 & 0 & 1 / 10 & 0 \\ 0 & 0 & 0 & 0\end{array}\right)$ and $U^{D} \geq 0$. So $A=U-V$ is an index-proper regular splitting. Also $A^{D}=\left(\begin{array}{cccc}0 & 0 & 1 / 25 & 0 \\ 0 & 1 / 5 & 0 & 0 \\ 0 & 0 & 1 / 5 & 0 \\ 0 & 0 & 0 & 0\end{array}\right) \geq 0$ and $\rho\left(U^{D} V\right)=1 / 2<1$

The next result shows the non-negativity of Drazin inverse under a sufficient condition.

Theorem 3.4. Let $A=U-V$ be an index-proper regular splitting of $A$. If $\rho\left(U^{D} V\right)<1$, then $A^{D} \geq 0$.

Proof. Suppose that $\rho\left(U^{D} V\right)<1$. By Theorem 2.6, $I-U^{D} V$ is nonsingular and $\left(I-U^{D} V\right)^{-1} \geq 0$. Then the expression $A^{D}=\left(I-U^{D} V\right)^{-1} U^{D}$ implies $A^{D} \geq 0$ as $U^{D} \geq 0$.

Similarly, we propose another extension of weak regular splitting called indexproper weak regular splitting. 
Definition 3.5. A splitting $A=U-V$ is called index-proper weak regular splitting if it is an index-proper splitting satisfying the conditions $U^{D} \geq 0$ and $U^{D} V \geq 0$.

The example given below is an index-proper weak regular splitting but not an index-proper regular splitting.

Example 3.6. Let $A=\left(\begin{array}{ccc}4 & -2 & 0 \\ -6 & 5 & 0 \\ 0 & 0 & 0\end{array}\right)=\left(\begin{array}{ccc}4 & -3 & 0 \\ -6 & 8 & 0 \\ 0 & 0 & 0\end{array}\right)-\left(\begin{array}{ccc}0 & -1 & 0 \\ 0 & 3 & 0 \\ 0 & 0 & 0\end{array}\right)=$ $U-V$. Then $R(A)=R(U)$ and $N(A)=N(U)$. Also, $U^{\#}$ exists $\left(\right.$ as $\left.r(U)=r\left(U^{2}\right)\right)$ and $U^{\#}=\left(\begin{array}{ccc}8 / 14 & 3 / 14 & 0 \\ 6 / 14 & 4 / 14 & 0 \\ 0 & 0 & 0\end{array}\right)$, and $U^{\#} V=\left(\begin{array}{ccc}0 & 1 / 14 & 0 \\ 0 & 6 / 14 & 0 \\ 0 & 0 & 0\end{array}\right)$. So, $A=U-V$ is an index-proper weak regular splitting but not an index-proper regular splitting since $V \nsupseteq 0$. (Note that in this case index of both $A$ and $U$ are 1.)

For an index-proper weak regular splitting of a Drazin monotone matrix, we have the following convergence theorem. The proof follows similar steps as in the proof of Theorem 3, [2].

Theorem 3.7. Let $A=U-V$ be an index-proper weak regular splitting with $N\left(A^{k}\right) \subseteq N(V)$. If $A$ is Drazin monotone, then $\rho\left(U^{D} V\right)<1$.

Proof. Let $S_{p}=\sum_{j=0}^{p}\left(U^{D} V\right)^{j}$ for each positive integer $p$. Then $S_{p} U^{D} A=$ $S_{p}\left[U^{D}(U-V)\right]=S_{p}\left(U^{D} U-U^{D} V\right)=U^{D} U-\left(U^{D} V\right)^{p+1}$ using the fact $V U^{D} U=V$ which again follows from the condition $N\left(A^{k}\right) \subseteq N(V)$. Now multiplying by $A^{D}$, we have $S_{p} U^{D} A A^{D}=S_{p} U^{D} U U^{D}=S_{p} U^{\bar{D}}=U^{D} U A^{D}-\left(U^{D} V\right)^{p} A^{D}=$ $A^{D}-\left(U^{D} V\right)^{p} A^{D}=\left[I-\left(U^{D} V\right)^{p}\right] A^{D}$ since $A A^{D}=U U^{D}$. Then $A^{D}-S_{p} U^{D}=$ $\left(U^{D} V\right)^{p} A^{D} \geq 0$ as $A^{D} \geq 0$ and $U^{D} V \geq 0$ (follows from the definition of indexproper weak regular splitting). Let $l \in \mathbb{R}_{+}^{n}$. Then $A^{D} l-S_{p} U^{D} l \in \mathbb{R}_{+}^{n}$. Let $t=A^{D} l$ and $s_{p}=S_{p+1} U^{D} l$. So $t$ and $s_{i}, i=0,1,2, \cdots$ satisfy Lemma 2.7 , and we then have $\lim _{i \rightarrow \infty}\left(s_{i}-s_{i-1}\right)=\lim _{i \rightarrow \infty}\left(U^{D} V\right)^{i} U^{D} l=0$. Since $U^{D} V \geq 0$ so there exist $y \in \mathbb{R}_{+}^{n}$ such that $U^{D} V y=\rho\left(U^{D} V\right) y$. So $y \in R\left(U^{D}\right)$ and then $y=U^{D} x$ for some $x \in \mathbb{R}^{n}$. Again, the fact $\mathbb{R}^{n}=\mathbb{R}_{+}^{n}+\left(-\mathbb{R}_{+}^{n}\right)$, implies $x=l_{1}-l_{2}$ for some $l_{1}, l_{2} \in \mathbb{R}_{+}^{n}$. Then for each positive integer $i,\left[\rho\left(U^{D} V\right)\right]^{i} y=\left(U^{D} V\right)^{i} y=$ $\left(U^{D} V\right)^{i} U^{D} x=\left(U^{D} V\right)^{i} U^{D} l_{1}-\left(U^{D} V\right)^{i} U^{D} l_{2}$ so that the sequence $\left[\rho\left(U^{D} V\right)\right]^{i}$ converges to 0 . Thus $\rho\left(U^{D} V\right)<1$.

The Example 3.6 illustrates the above theorem. One can see that $N(A) \subseteq$ $N(V)$ and $\rho\left(U^{\#} V\right)=6 / 14<1$ in the same example. Again, the condition $\rho\left(U^{D} V\right)<1$ yields $A^{D} \geq 0$ proceeding as in proof of Theorem 3.4, and the result is stated as follows.

Theorem 3.8. Let $A=U-V$ be an index-proper weak regular splitting of $A$. If $\rho\left(U^{D} V\right)<1$, then $A^{D} \geq 0$.

Climent and Perea, [7] first established the theory of multisplitting and its convergence results for rectangular matrices using the Moore-Penrose inverse. 
Motivated by their work, we now introduce similar theory for singular square matrices using the Drazin inverse. Let $A=U-V$ be an index-proper splitting of $A \in \mathbb{R}^{n \times n}$ and let $U=R-S$ be an index-proper splitting of $U$. If we consider $q$ inner iterations with the index-proper splitting $U=R-S$, then the two-stage iterative method is

$$
\begin{gathered}
x^{i+1}=\left(R^{D} S\right)^{q} x^{i}+\sum_{j=0}^{q-1}\left(R^{D} S\right)^{j} R^{D}\left(V x^{i}+b\right) \\
\text { So } x^{i+1}=\left(R^{D} S\right)^{q} x^{i}+\sum_{j=0}^{q-1}\left(R^{D} S\right)^{j} R^{D} V x^{i}+\sum_{j=0}^{q-1}\left(R^{D} S\right)^{j} R^{D} b \\
=\left(\left(R^{D} S\right)^{q}+\sum_{j=0}^{q-1}\left(R^{D} S\right)^{j} R^{D} V\right) x^{i}+\sum_{j=0}^{q-1}\left(R^{D} S\right)^{j} R^{D} b \\
=G x^{i}+c,
\end{gathered}
$$

where $G=\left(R^{D} S\right)^{q}+\sum_{j=0}^{q-1}\left(R^{D} S\right)^{j} R^{D} V$ and $c=\sum_{j=0}^{q-1}\left(R^{D} S\right)^{j} R^{D} b$. Now, using the above two-stage iterative method, we have the following theorem.

Theorem 3.9. Let $A=U-V$ be an index-proper regular splitting of a Drazin monotone matrix $A$ with $R(V) \subseteq R\left(A^{k}\right)$ and $U=R-S$ be an index-proper weak regular splitting with $N\left(U^{k}\right) \subseteq N(S)$. Then, the stationary two-phase iterative method (3.1) converges to the solution $A^{D} b$ of the system $A x=b$ for any initial vector $x^{(0)}$.

Proof. Since $A=U-V$ is an index-proper regular splitting with $R(V) \subseteq R\left(A^{k}\right)$, so $U U^{D} V=V, R^{D} U=R^{D} R-R^{D} S$ and $R^{D} R=U^{D} U$. From equation (3.1), we now have

$$
\begin{aligned}
G & =\left(R^{D} S\right)^{p}+\sum_{j=0}^{p-1}\left(R^{D} S\right)^{j} R^{D} V \\
& =\left(R^{D} S\right)^{p}+\sum_{j=0}^{p-1}\left(R^{D} S\right)^{j} R^{D} U U^{D} V \\
& =\left(R^{D} S\right)^{p}+\sum_{j=0}^{p-1}\left(R^{D} S\right)^{j}\left(R^{D} R-R^{D} S\right) U^{D} V \\
& =\left(R^{D} S\right)^{p}+\left(R^{D} R-\left(R^{D} S\right)^{p}\right) U^{D} V \\
& =\left(R^{D} S\right)^{p}+R^{D} R U^{D} V-\left(R^{D} S\right)^{p} U^{D} V \\
& =\left(R^{D} S\right)^{p}+U^{D} U U^{D} V-\left(R^{D} S\right)^{p} U^{D} V \\
& =\left(R^{D} S\right)^{p}+U^{D} V-\left(R^{D} S\right)^{p} U^{D} V \\
& =\left(R^{D} S\right)^{p}+\left(I-\left(R^{D} S\right)^{p}\right) U^{D} V \\
& =I-I+\left(R^{D} S\right)^{p}+\left(I-\left(R^{D} S\right)^{p}\right) U^{D} V \\
& =I-\left(I-\left(R^{D} S\right)^{p}\right)\left(I-U^{D} V\right) \\
& =I-\sum_{j=0}^{p-1}\left(R^{D} S\right)^{j}\left(I-R^{D} S\right)\left(I-U^{D} V\right)
\end{aligned}
$$


Since the splitting $A=U-V$ is index-proper regular, so $U^{D} \geq 0$. Again, $U=R-S$ is index-proper weak regular. Also, $A^{D} \geq 0$. So, by Theorems 3.2 and 3.7, we have $\rho\left(U^{D} V\right)<1$ and $\rho\left(R^{D} S\right)<1$. Using Theorem 2.6, we then get $\left(I-U^{D} V\right)^{-1} \geq 0$ and $\left(I-R^{D} S\right)^{-1} \geq 0$. Now, for any $y \geq 0, x=$ $\left(I-U^{D} V\right)^{-1}\left(I-R^{D} S\right)^{-1} y \geq 0$. Hence,

$$
0 \leqslant G x=x-\sum_{j=0}^{p-1}\left(R^{D} S\right)^{j} y<x .
$$

Then, by Lemma 2.5, $\rho(G)<1$.

Let us now introduce the definition of an index-proper multi splitting.

Definition 3.10. The triplet $\left(U_{l}, V_{l}, W_{l}\right)_{l=1}^{p}$ is called an index-proper multisplitting of $A$ if $A=U_{l}-V_{l}$, for $l=1,2,3, \cdots p$, is an index-proper splitting and each $W_{l}$, is a non-negative and diagonal matrix such that $\sum_{l=1}^{p} W_{l}=I$, where $I$ is the identity matrix of order $n$.

A multisplitting is an index-proper regular or an index-proper weak regular splitting, if each one of the splitting is an index-proper regular or an indexproper weak regular splitting, respectively. If $\left(U_{l}, V_{l}, W_{l}\right)_{l=1}^{p}$ is an index-proper multisplitting of $A$, then we consider the iterative scheme

$$
x^{i+1}=H x^{i}+d, i=0,1,2, \cdots,
$$

where $H=\sum_{l=1}^{p} W_{l} U_{l}^{D} V_{l}$ and $d=\sum_{l=1}^{p} W_{l} U_{l}^{D} b$. The following result is helpful before proving the convergence criteria of the above proposed splitting.

Lemma 3.11. Let $\left(U_{l}, V_{l}, W_{l}\right)_{l=1}^{p}$ be an index-proper weak regular multisplitting of $A$ with $R\left(V_{l}\right) \subseteq R\left(A^{k}\right)$. Then

(a) $H \geq 0$ and $H^{j} \geq 0$, for $j=0,1, \cdots$,

(b) $\sum_{l=1}^{p} W_{l} U_{l}^{D} A=(I-H) A^{D} A$,

(c) $\left(I+H+H^{2}+\cdots+H^{n}\right)(I-H)=I-H^{n+1}$.

Proof. (a): Since the matrix $H=\sum_{l=1}^{p} W_{l} U_{l}^{D} V_{l}$ and given multisplitting is an index-proper weak regular of $A$, then for each $l=1,2, \cdots p, U_{l}^{D} \geq 0, U_{l}^{D} V_{l} \geq 0$ and $W_{l}$ is non-negative diagonal matrix. So $H \geq 0$. Hence $H^{j} \geq 0$, for $j=$ $0,1, \cdots$.

(b): The condition $R\left(V_{l}\right) \subseteq R\left(A^{k}\right)$ implies $U_{l} U_{l}^{D} V_{l}=V_{l}=U_{l}^{D} U_{l} V_{l}$ and the indexproper splitting of $A$ gives $U_{l}^{D} U_{l}=A^{D} A$ for $l=1,2, \cdots, p$. Since $A$ can be written as

$$
A=U_{l}-V_{l}=U_{l}\left(I-U_{l}^{D} V_{l}\right), l=1,2, \cdots, p,
$$


then

$$
\begin{aligned}
\sum_{l=1}^{p} W_{l} U_{l}^{D} A & =\sum_{l=1}^{p} W_{l} U_{l}^{D} U_{l}\left(I-U_{l}^{D} V_{l}\right) \\
& =\sum_{l=1}^{p} W_{l}\left(U_{l}^{D} U_{l}-U_{l}^{D} U_{l} U_{l}^{D} V_{l}\right) \\
& =\sum_{l=1}^{p} W_{l}\left(U_{l}^{D} U_{l}-U_{l}^{D} V_{l} U_{l}^{D} U_{l}\right) \\
& =\sum_{l=1}^{p} W_{l}\left(I-U_{l}^{D} V_{l}\right) U_{l}^{D} U_{l} \\
& =\left(I-\sum_{l=1}^{p} W_{l} U_{l}^{D} V_{l}\right) A^{D} A \\
& =(I-H) A^{D} A .
\end{aligned}
$$

(c): Since $H^{j} \geq 0$ for each $j$, then the sum $\left(I+H+H^{2}+\cdots+H^{n}\right)(I-H)=$ $I-H^{n+1}$.

Theorem 3.12. Let $\left(U_{l}, V_{l}, W_{l}\right)_{l=1}^{p}$ be an index-proper weak regular multisplitting of $A$. Then, the iterative method (3.2) converges to the solution $A^{D} b$ of system $A x=b$ for any initial vector $x^{(0)}$.

Proof. Using Lemma 3.11, and the properties $U_{l}^{D} U_{l} U_{l}^{D}=U_{l}^{D}$ and $U_{l} U_{l}^{D}=A A^{D}$ for $l=1,2, \cdots, p$, and $A^{D} A A^{D}=A^{D}$, we get

$$
\begin{aligned}
0 & \leq\left(I+H+H^{2}+\cdots+H^{n}\right) \sum_{l=1}^{p} W_{l} U_{l}^{D} \\
& =\left(I+H+H^{2}+\cdots+H^{n}\right) \sum_{l=1}^{p} W_{l} U_{l}^{D} U_{l} U_{l}^{D} \\
& =\left(I+H+H^{2}+\cdots+H^{n}\right) \sum_{l=1}^{p} W_{l} U_{l}^{D} A A^{D} \\
& =\left(I+H+H^{2}+\cdots+H^{n}\right)(I-H) A^{D} A A^{D} \\
& =\left(I+H+H^{2}+\cdots+H^{n}\right)(I-H) A^{D} \\
& =\left(I-H^{n+1}\right) A^{D} \leq A^{D}
\end{aligned}
$$

So, the elements of $H^{n}$ are bounded. Hence, $H$ is convergent, i.e., iterative method (3.2) converges to the solution of system $A x=b$ for initial vector $x^{(0)}$.

\section{Conclusions}

We also note that a few results (Theorem 3.4 and 3.8) of this paper can be applied to find whether a square singular matrix has a non-negative Drazin inverse or not. In fact, for a square singular system of linear equations $A x=b$, where $x, b \in R^{n}$ and $A \in R^{n \times n}$, the solution $A^{D} b$ lies in the Krylov subspace 
of $(A, b)$, i.e., $K_{s}(A, b)=\operatorname{span}\left\{b, A b, A^{2} b, \ldots, A^{s-1} b\right\}$. The connection between Drazin inverse and Krylov subspace is now recalled. $A^{D} b$ is a solution of $A x=b$, $b \in R\left(A^{k}\right)$ if and only if $A x=b$ has a solution in $K_{n}(A, b)$, where $n$ is the order of the matrix $A$. Finally, we close the last section with a result that has a possible application to numerical analysis for faster computation of solution $A^{D} b$.

Besides these, we would now like to recall some works in connection with the present work. Cui et al., [16] and Lin et al., [12] studied properties of convergence and quotient convergence of iterative methods for solving consistent singular linear systems with index one. Cui et al., [16] also applied the same theory to multisplittings. Note that both their works deal with matrices of index one while we do not have any restriction on index. However, Wei and $\mathrm{Wu}$, [18] presented another extension of proper splittings called a $(T, S)$-splitting which is also more general than index-proper splittings. We now reproduce the same splitting. Let $A$ be a real rectangular $m \times n$ matrix with rank $r$. Let $T$ be a subspace of $\mathbb{R}^{n}$ of dimension $s \leq r$, and $S$ be a subspace of $\mathbb{R}^{m}$ of dimension $m-s$. A splitting $A=U-V$ is called a $(T, S)$ splitting $([18])$ if $R(A)=A T$ and $N(A)=\left(A^{*} S^{\perp}\right)^{\perp}$. When $T=R\left(A^{*}\right)$ and $S=N\left(A^{*}\right)$, this reduces to a proper splitting of $A$. (We used $B^{*}$ to denote transpose of $B$ for notational simplicity.) If $A$ is square, and $T=R\left(A^{k}\right)$ and $S=N\left(A^{k}\right)$ where $k$ is the index of $A$, then a $(T, S)$ splitting of $A$ reduces to an index splitting of $A$. Wei and Wu, [18] then considered the iterative scheme

$$
y^{i+1}=U_{T, S}^{(1,2)} V y^{i}+U_{T, S}^{(1,2)} b, \quad i=0,1,2, \cdots
$$

and

$$
Y^{i+1}=U_{T, S}^{(1,2)} V Y^{i}+U_{T, S}^{(1,2)}, \quad i=0,1,2, \cdots
$$

to compute a solution $A_{T, S}^{(2)} b$ of the system $A x=b$ where $A \in \mathbb{R}^{m \times n}$ and $b \in \mathbb{R}^{m}$, and the generalized inverse $A_{T, S}^{(2)}$, where $G_{T, S}^{(1,2)}$ denotes a $\{1,2\}$-inverse of $G$ whose range is $T$ and null space is $S$. They studied the convergence theory for the above schemes. Different subclasses of the above splittings, namely, $(T, S)$-regular and $(T, S)$-weak regular can be proposed, and then the convergence theory can also be studied.

Acknowledgement. The authors would like to thank the reviewers for their helpful suggestions.

\section{REFERENCES}

1. A. Berman and R.J. Plemmons, Nonnegative Matrices in the Mathematical Sciences, SIAM, Philadelphia, 1994.

2. A. Berman and R.J. Plemmons, Cones and iterative methods for best square least squares solutions of linear systems, SIAM J. Numer. Anal. 11 (1974), 145-154.

3. A. Berman and R.J. Plemmons, Matrix group monotonicity, Proc. Amer. Math. Soc. 46(1974), 355-359.

4. A. Berman and R.J. Plemmons, Monotonicity and the generalized inverse, SIAM J. Appl. Math. 22 (1972), 155-161.

5. D. Mishra and K.C. Sivakumar, A dominance notion for singular matrices with applications to nonnegative generalized inverses, Linear and Multilinear Algebra 60 (8) (2012), 911920 . 
6. I. Marek and D.B. Szyld, Comparison theorems for weak splittings of bounded operators, Numer. Math. 58 (1990), no. 4, 387-397.

7. J.-J. Climent and C. Perea, Iterative methods for least square problems based on proper splittings, J. Comput. Appl. Math. 158 (2003), 43-48.

8. L. Collatz, Functional Analysis and Numerical Mathmatics, Academic press, New YorkLondon, 1966.

9. L. Jena and D. Mishra, $B_{D}$-splittings of matrices, Linear Algebra Appl. 437 (2012), no. 4, $1162-1173$.

10. L. Jena, D. Mishra and S. Pani, Convergence and comparison theorems for double splittings of matrices, Calcolo 51 (2014), no. 1, 141-149.

11. L. Jena and S. Pani, Index-range monotonicity and index proper splittings of matrices, Numer. Algebra Control Optim. 3 (2013), no 2, 379-388.

12. L. Lin, Y. Wei and N. Zhang, Convergence and quotient convergence of iterative methods for solving singular linear equations with index one, Linear Algebra Appl. 430 (2009), $1665-1674$.

13. O.L. Mangasarian, Characterization of real matrices of monotone kind, SIAM Rev. 10 (1968), 439-441.

14. R.S. Varga, Matrix Iterative Analysis, Springer-Verlag, Berlin, 2000.

15. W.C. Pye, Nonnegative Drazin inverses, Linear Algebra Appl. 30 (1980), 149-153.

16. X. Cui, Y. Wei and N. Zhang, Quotient convergence and multi-splitting methods for solving singular linear equations, Calcolo 44 (2007), no. 1, 21-31.

17. Y. Wei, Index splitting for the Drazin inverse and the singular linear system, Appl. Math. Comput. 95 (1998), no.2-3, 115-124.

18. Y. Wei and $\mathrm{H}$. Wu, $(T, S)$ splitting methods for computing the generalized inverse $A_{T, S}^{(2)}$ and rectangular systems, Int. J. Comput. Math. 77 (2001), no.3, 401-424.

1 Department of Mathematics, Utkal University, Vanivihar, Bhubaneswar 751 004, INDIA.

E-mail address: alekha.math@gmail.com

2 School of Basic Sciences, Indian Institute of Technology Bhubaneswar, BHUBANESWAR - 751 013, INDIA.

E-mail address: litumath@gmail.com 